\title{
Índice de vulnerabilidad estructural ante los efectos de remoción en masa en edificaciones de mampostería basado en conjuntos difusos
}

\section{Structural vulnerability index to the effects of landslides in masonry buildings based on fuzzy sets}

\author{
Esperanza Maldonado Rondón*1, Gustavo Chio Cho* \\ * Universidad Industrial de Santander. COLOMBIA
}

\begin{abstract}
Fecha de recepción: 25/ 05/ 2011 Fecha de aceptación: 15/ 12/ 2011 Resumen
\end{abstract}


Consequently, it is important to work identifying the potential areas which would be affected by an effective natural disaster threat as well as to provide a better construction planning for future buildings construction, in order to achieve a less vulnerable city. This would only be possible if a vulnerability study for the cities is developed.

Generally, experience has demonstrated that action is taken after disasters have occurred. Most existing vulnerability, threads and risks studies due to landslides have been encouraged after tragedies have taken place in the past and this practice must be avoided. It is necessary to develop complete and fully supported studies, not conditioned to time limitations urging to deliver prompt results and proposing immediate measures, which are unaware of the real problem intended to solve. Therefore, effective caution and improvement measures will be only offered to the community with a clear planning on how buildings are and how they respond to landslide effects.

Therefore, the main purpose of this research is to introduce a new methodology to estimate building vulnerability of a given area to landslide effects. The model is based on the assessment of the most relevant characteristics of a masonry structure, which may affect its vulnerability faced to abovementioned effects. Quoted characteristics have been determined by selecting fourteen parameters. Parameters were classified in accordance to three or four quality standards, which were assigned with a specific vulnerability degree. Besides, each parameter was assigned with an importance value. These vulnerability degrees and weight value were identified by expert opinions.

Since the proposed model involves expert opinions and its further application is based on the responses to a form which classifies each parameter; it was decided to employ fuzzy sets techniques. Among fuzzy sets diffuse factorial was used, as a means of calculation for vulnerability index. Such index is the relation between vulnerability degree of each parameter and its importance value. 


\section{Vulnerability estimation models}

Nowadays there are different proposed methodologies to estimate building vulnerability to landslide actions. However, a great deal employs historical data or they are based on the experience obtained from past events. Then, in areas lacking of past damages records, construction and adaptation of such methodologies is not direct. Therefore, it is important to develop vulnerability estimation models depending on the buildings own characteristics and the places were they will be implemented.

Uzielli et al. (2008) introduce a quantitative vulnerability estimation method based on a probabilistic approach. They start by associating vulnerability to the lack of inherent capacity of the structure to preserve its physical integrity and functional capacity during the occurrence of a landslide effect and, they estimate that this mainly depends on the structure characteristic and its maintenance conditions. So they define vulnerability in function of structural typology and maintenance conditions.

Leone et al. (1996) propose a model to evaluate damages due to landslide phenomena, based on damage matrixes built from statistics on past records. To do so, they introduce the concept of structure vulnerability in function of the structure type, age and its strength by assigning a value from 0 to 1 .

Finlay et al. (1997) depict an example of vulnerability estimation by assigning a value from 0 to 1 to vulnerability index based on historical records, without considering several components affecting vulnerability. Values are assigned in accordance with historical experience available from threat management.

\section{Model parameters}

The proposed model is based on the assessment of 14 building parameters and their closest surroundings, which are considered of major influence on a masonry building vulnerability faced to landslide effects. Each one of them were assigned with three or four quality standards (A, B, C y D), as follows: 
Parameter 1 - Structural system

A. Squared Masonry in every floor plan

B. Reinforced masonry in every floor plan

C. Squared masonry not in every floor plan

D. Masonry which only has supporting beams, without columns, columns without supporting beams

E. Non-squared masonry in every floor plant

Parameter 2 - quality of load-bearing structure

A. High quality masonry, with uniform pieces properly bonded

B. High quality masonry, with not quite uniform pieces but properly bonded

C. Low quality masonry with uniform and non-uniform pieces, properly bonded

D. High and low quality masonry, with non-uniform pieces or improperly bonded

Parameter 3 - Structural strength

A. Building with a value of $\alpha<1$. ( $a$ is the capacitydemand relation to movements in the building foundation)

B. Building with a value of $0.6=\alpha<1$.

C. Building with a value of $0.4=\alpha<0.6$.

D. Building with a value of $\alpha<0.4$.

Parameter 4 - Height lay out

A. Building with $-\Delta M / M<10 \%$ or only one storey building ( $\Delta \mathrm{M}$ is the maximum mass variation between two storey, being (-) for decrease and (+) for increase; $M$ is the mass of lower storey.

B. $10 \% \leq-\Delta \mathrm{M} / \mathrm{M}<20 \%$.

C. $-\Delta M / M>20 \%$.

D. $+\Delta M / M>0$.

Parameter 5 - Building location

A. Building shall meet one of the following conditions:

1. Building placed on a gradient lower than $30^{\circ}$ or beyond a slope higher than $30^{\circ}$, fulfilling required isolation distances already established. 
2. Building placed on a hill top with land gradient between $30^{\circ}$ and $45^{\circ}$, on a slope lower than 5 meters high, but counting with a rigid-structure-retaining wall, which height is equal or higher than the slope total height.

3. Building placed under the base or slope foundation, with a total height lower than 5 meters, counting with a rigid-structure-retaining wall, which height is equal or higher than the slope total height.

B. Not fulfilling A either C.

C. Building having one of the following characteristics:

1. Building placed on the slope with natural gradient lower than $30^{\circ}$ or out of its scope, but not fulfilling required isolation distances

2. Built on cross sections

3. Placed on a cross section of a natural land gradient higher than $30^{\circ 0}$

4. Placed on a back-filled hillside

Parameter 6 - Foundation location

A. Foundation is located at the same benchmark. Lack of unbalanced pressure due to back-filling.

B. Maximum differences among foundation benchmarks are lower than $\mathbf{1}$ meter, with lack of unbalanced pressure due to backfill or; because the foundation is located at the same benchmark but there is presence of unbalanced pressure due to backfill.

C. Maximum differences among foundation benchmarks are lower than $\mathbf{1}$ meter, with presence of unbalanced pressure due to backfill.

D. Maximum differences among foundation benchmarks are higher than 1 meter

Parameter 7 - Foundation Types

A. Piles

B. Continuous footing

C. Building on foundation slab

D. Building is supported on slender elements laying on deep footings

E. Building is supported on slender elements laying on the ground-surface footing 
Parameter 8 - Type of soil

A. Building founded on rocky or rigid soil

B. Building founded on back-fills

Parameter 9 - Vegetation existing on the area

A. Building is in an area where natural surrounding has been maintained

B. Building is in an area where natural surrounding has not been maintained and little original vegetation is kept.

C. The area where the building has been constructed has suffered a major changes and a discriminated cleaning has been executed.

Parameter 10 - Retaining walls

A. Building does not require retaining walls. If needed they shall meet the following:

a. Walls must be designed to endure soil and water actions

b. Walls must be founded on rocky or firm soil.

c. Walls must have a proper drainage system behind vertical wall (Suarez, 2009).

d. Back-fill materials must be clean and compact in accordance with engineering regulations.

B. Building not fulfilling A or C.

C. Building requires a retaining wall but does not count with it. Building requires structural walls but they:

a. They are improperly constructed using loose-fitting or under-compacted material

b. Back-fill contains logs, trees, vegetation, unstable rocks, construction waste material, etc.

c. A proper drainage system is not available

Parameter 11 - Drainage system

A. Meet the following

a. Sewage system is available

b. There are surface drainages for rainy waters which discharge into a sewage system.

c. Storage tanks are airtight and properly founded.

B. Not fulfilling A or C. 
C. Fulfilling the following:

a. Rainy waters collected by the building roof are delivered directly to the slope.

b. Sewage waters are delivered directly to the slope.

c. There are no surface drainages available.

d. Storage tanks are not airtight and they are not properly founded.

Parameter12 - Handling of waste materials

A. There is an adequate waste collection system

B. Waste collection system is not appropriate or waste material is directly dumped to the hillside.

Parameter 13 - Surrounding conservation conditions

A. The area has clean drainage systems. There are no miss-matched joints in the sewage system or leakage in the supply piping either. Water leakage is not observed on the soil.

B. Does not meet A either C.

C. The area counts with a clean drainage system. Furthermore, there is at least one of the following aspects:

a.There are broken joints alongside the sewage system b.There are leakages in the supply piping c.There are visible leakages on the soil

Parameter 14 - Building Preservation Conditions

A. Walls in good conditions, non visible crackings.

B. Walls having non-extended crackings.

C. Walls having medium size crackings, ranging from 2 up to 3 millimeters width. Building that does not have crackings but it is characterized by a poor masonry preservation conditions.

D. Walls have a major material damage or have crackings higher than 3 millimeters width. 
Each parameter has, in turn, an associated importance value in the vulnerability estimation of the whole structure.

\section{Definition of the vulnerability index model}

In this paper vulnerability is quantitatively defined as the susceptibility of the elements to suffer damages due to landslides effects. The proposed model is a complete description of required information to obtain a quantitative expression of vulnerability, called Vulnerability Index. This index relates parameters vulnerability degrees mostly affecting building vulnerability and their importance values in the total vulnerability estimation. Selected parameters are associated in accordance with the structures own characteristics, their location and surrounding factors. Besides, the input associated to structural aspects can be direct or indirect, see Figure 1.

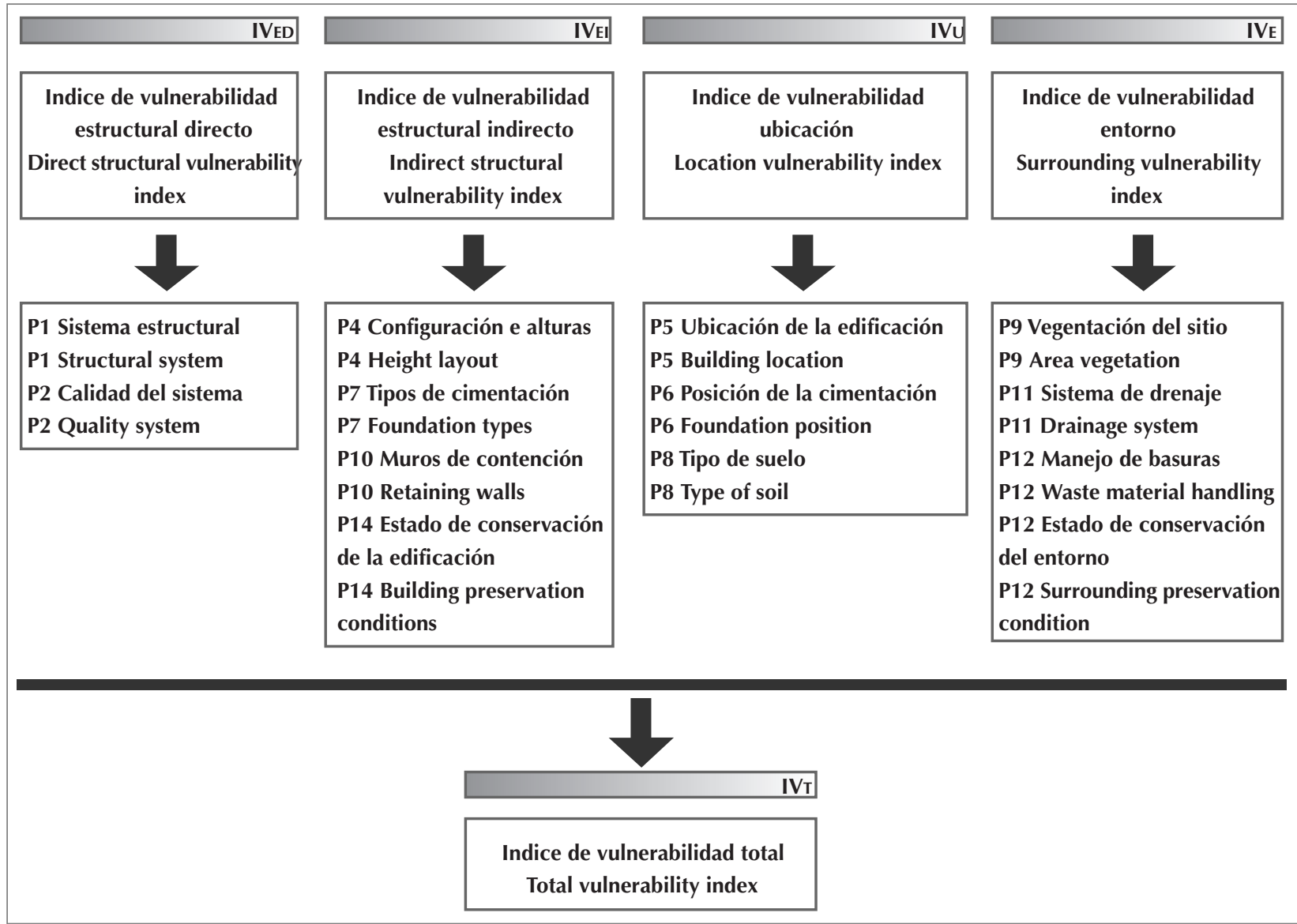

Figura 1. Esquema de la definición del modelo del índice de vulnerabilidad Figure 1. Definition lay out of vulnerability index model 
The model is based on the estimation of a total vulnerability index $\left(\mathrm{IV}_{\mathrm{T}}\right)$, which is the input of four estimated partial vulnerability indexes. One is associated with direct aspects $\left(I_{V} E_{D}\right)$, other with indirect structural aspects $\left(I V_{E I}\right)$, other with building locations $\left(I V_{U}\right)$ and finally another associated with surroundings aspects $\left(\mathrm{IV}_{\mathrm{E}}\right)$. Each one of them has a range from 0 (non vulnerable) to 1 (completely vulnerable). See Figure 1.

Each index is estimated from qualifications of different quality conditions on selected parameters (A, B, C and D) named $K_{I}$. The importance values of each parameter in the total vulnerability determination of the building are named $W_{i}$. And values $K_{I}$ and $W_{i}$ were analyzed under fuzzy sets because of their definition ambiguity (Zadeh, 1965; Chakroborty and Kikuchi, 1990; Chao and Ayyub, 1995; Maldonado 2007). Diffuse arithmetic (Bandemer y Gottwald, 1996; Tee, et. al., 1988) was employed to relate qualifications of each parameter $\left(K_{I}\right)$ and their respective importance values $\left(W_{i}\right)$. The proposed method to combine diffuse information - of uneven importance or weights - was diffuse factorial, which was used to determine vulnerability index, expressed as:

$$
\begin{gathered}
I . V .=1-\prod_{i=1}^{n}\left(1-\varepsilon_{i}\right) \\
\varepsilon_{i}=K_{i} * W_{i}
\end{gathered}
$$

Where I.V. is the vulnerability index; $\varepsilon_{i}$ is $i$-th of $n$ vulnerability factor (each one defined in a range of [0.1]); $K_{i}$ is the vulnerability degree of i parameter category $i$; $W_{i}$ is the importance associated to parameter $i$ in relation to the other parameters and; $n$ is the number of parameters in the model.

Therefore, each index will be determined as follows:

$$
\text { I. } V_{E D}=1-\prod_{i=1}^{3}\left(1-\varepsilon_{i}\right)
$$

Parameters associated to this index are structural system, quality and structural strength.

$$
\text { I. } V_{E I}=1-\prod_{i=1}^{4}\left(1-\varepsilon_{i}\right)
$$


This index will include parameters associated with height lay out, type of foundation, retaining walls and preservation conditions.

$$
\text { I. } V_{U}=1-\prod_{i=1}^{3}\left(1-\varepsilon_{i}\right)
$$

This index will consider parameters associated with location, foundation level and the type of soil where building is constructed.

$$
\text { I. } V_{L}=1-\prod_{i=1}^{4}\left(1-\varepsilon_{i}\right)
$$

This index considers the parameters related to building surroundings, which are directly related to them, whose affectation can actually damage vulnerability. Consequently, parameters associated with this group are: site vegetation, drainage system, waste material control and surrounding preservation.

In order to estimate total vulnerability index, the higher value among direct structural vulnerability index $I V_{E D}$ and, location index $I_{V U}$ are considered in the first place. Then the remaining vulnerability indexes are accounted and they are averaged out. Finally, these values are replaced in Equation 7.

$$
I . V_{T}=I V_{E D o U}\left[1+0.2 \frac{I V_{E I}+I V_{E}}{2}\right] \leq 1
$$

Total vulnerability index will always be lower or equal to 1 .

\section{Algorithm used for the calculation of vulnerability index}

Calculation of each vulnerability index, including total index, was developed by means of the use of fuzzy sets, as follows.

1) Translation of qualification variables of fourteen-quality conditions parameters into fuzzy sets is made by means of a definition of the corresponding membership functions based on expert opinions.

2) Conversion of importance variables of each parameter into fuzzy sets by employing membership functions based on expert opinions. 
3) Permutation of qualification variables and fuzzy importance variables to obtain fuzzy sets representing vulnerability indexes expressed in Equations 2 to 7 . Such permutation provides fuzzy set results called indirect structural, direct structural, location, surroundings and total vulnerability indexes.

4) Transfiguration of the remaining fuzzy set, total vulnerability index into linguistic expression such as very low, low, medium, high or very high vulnerability. Transfiguration of a fuzzy set into natural linguistic expressions is a relatively simple process, which involves distance determination of the remaining fuzzy set to each fuzzy set representing the quoted linguistic expressions. The minimum distance between the remaining fuzzy set and the fuzzy sets representing linguistic variables, is the linguistic expression associated with the vulnerability index.

5) Association of vulnerability index to the linguistic variable calculated from a non-fuzzy numerical value. It was developed by choosing a numeric value from function gravity center of membership function resulting from Equation 7.

\section{Employed linguistic variables}

For the definition of vulnerability degrees of this model's parameters and importance values obtained from fuzzy sets, it was necessary to accurately define the linguistic variables to describe different degrees of vulnerability and importance values. Such linguistic variables were employed to characterize each quality condition assigned to each parameter and; to define the importance of each parameter in the total structural vulnerability estimation. Therefore, natural linguistic variables selected to characterize vulnerability degrees were vey low, low, medium, high or very high. Each variable was represented by fuzzy sets as follows.

- Very low vulnerability =

$\{1|0,0| 0.2\}$

- Low vulnerability =

$\{0|0.15,1| 0.3,0 \mid 0.45\}$

- Medium vulnerability =

$\{0|0.35,1| 0.5,0 \mid 0.65\}$

- High vulnerability =

$\{0|0.55,1| 0.7,0 \mid 0.85\}$

- Very high vulnerability =

$\{0|0.8,1| 1\}$ 
Being:

$y=\left\{\mu_{(x)} \mid x\right\}$

Where $\mu_{(x)}$ indicates $x$ membership degree; defining $x$ as the universe of building vulnerability in fuzzy sets.

The graphical representation of membership functions of linguistic variables employed in this research are shown in Figure 2.

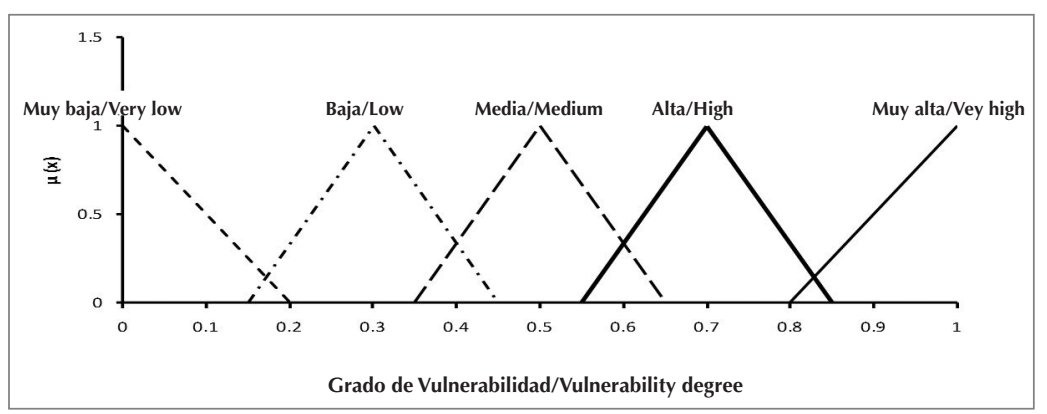

Figura 2. Funciones de pertenencia de las variables lingüísticas muy baja, baja, medio, alta y muy alta

Figure 2. Membership functions of linguistic variables vey low, low, medium, high or very high

\section{Quality and importance values of the model}

Membership functions of quality conditions and created importance values of parameters to be analyzed by the proposed model are presented in Tables 1 and 2 .

\section{Model application method}

For the application of this model, it is necessary to make an inventory including the buildings of the area under study. Such inventory consists of gathering the necessary information to assign a quality standard to each fourteen parameters of the model. Afterwards the information is stored by a SIG, which includes the vulnerability index calculation (UIS and CDMB, 2011). Finally by means of SIG data processing, a vulnerability mapping is obtained for the area under study. Such mapping identifies vulnerability degree of each building in the area. 
Tabla 1. Funciones de pertenencia para los valores de calidad de cada uno de los parámetros, $K_{i}$ Table 1. Membership functions for quality values of each $K_{i}$, parameter

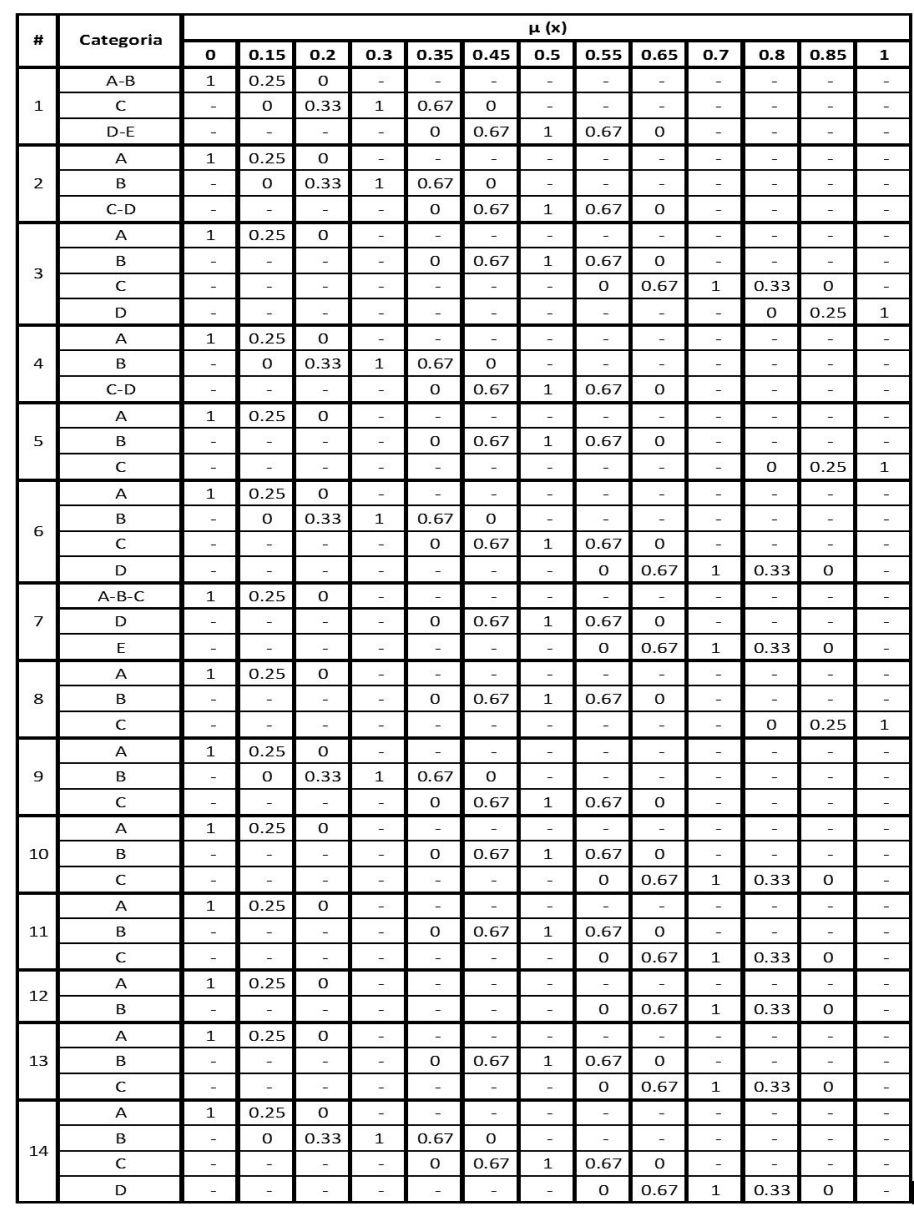

\# se refiere al número del parámetro \# refers to parameter number

Tabla 2. Funciones de pertenencia para los valores de importancia, $W_{i}$ Table 2. Membership functions for $W_{i}$ importance values

\begin{tabular}{|c|c|c|c|c|c|c|c|c|c|c|c|c|c|}
\hline \multirow{2}{*}{$\#$} & \multicolumn{13}{|c|}{$\mu(x)$} \\
\hline & 0 & 0.15 & 0.2 & 0.3 & 0.35 & 0.45 & 0.5 & 0.55 & 0.65 & 0.7 & 0.8 & 0.85 & 1 \\
\hline 1 & - & - & - & - & - & - & - & 0 & 0.67 & 1 & 0.33 & 0 & - \\
\hline 2 & - & - & - & - & 0 & 0.67 & 1 & 0.67 & 0 & - & - & - & - \\
\hline 3 & - & - & - & - & 0 & 0.67 & 1 & 0.67 & 0 & - & - & - & - \\
\hline 4 & - & - & - & - & 0 & 0.67 & 1 & 0.67 & 0 & - & - & - & - \\
\hline 5 & - & - & - & - & - & - & - & - & - & - & 0 & 0.25 & 1 \\
\hline 6 & - & - & - & - & 0 & 0.67 & 1 & 0.67 & 0 & - & - & - & - \\
\hline 7 & - & - & - & - & - & - & - & 0 & 0.67 & 1 & 0.33 & 0 & - \\
\hline 8 & - & - & - & - & - & - & - & 0 & 0.67 & 1 & 0.33 & 0 & - \\
\hline 9 & - & - & - & - & 0 & 0.67 & 1 & 0.67 & 0 & - & - & - & - \\
\hline 10 & - & - & - & - & - & - & - & 0 & 0.67 & 1 & 0.33 & 0 & - \\
\hline 11 & - & - & - & - & 0 & 0.67 & 1 & 0.67 & 0 & - & - & - & - \\
\hline 12 & - & 0 & 0.33 & 1 & 0.67 & 0 & - & - & - & - & - & - & - \\
\hline 13 & - & - & - & - & 0 & 0.67 & 1 & 0.67 & 0 & - & - & - & - \\
\hline 14 & - & - & - & - & - & - & - & 0 & 0.67 & 1 & 0.33 & 0 & - \\
\hline
\end{tabular}

\# se refiere al número del parámetro \# refers to parameter number 


\section{Model application}

Vulnerability index model was applied on a sector in Bucaramanga city, located in an area called El Porvenir, which has $\mathbf{1 . 1 1 0}$ houses having squared masonry in every storey that corresponds to $54.7 \%$, while the remaining $45.3 \%$ are squared structures in the first floor, but the following floors have square or semi-square structures. Most buildings are two-story buildings (54.5\%) and the remaining ones are: three-storey buildings (24.5\%), one-storey (20\%) and even four-storey buildings (1\%). They were built in the 80's and 90's, according to antiseismic regulations in force at that time. One-storey houses were delivered to owners who had the possibility to build a second floor. Over the years, most of them were enlarged into two and three storey houses, without following proper construction design patterns. The area is located on a slope and some houses are quite close to the slope gradient edge.

Then the inventory was made and respective vulnerability mapping calculations were obtained, which are presented in Figure 3.

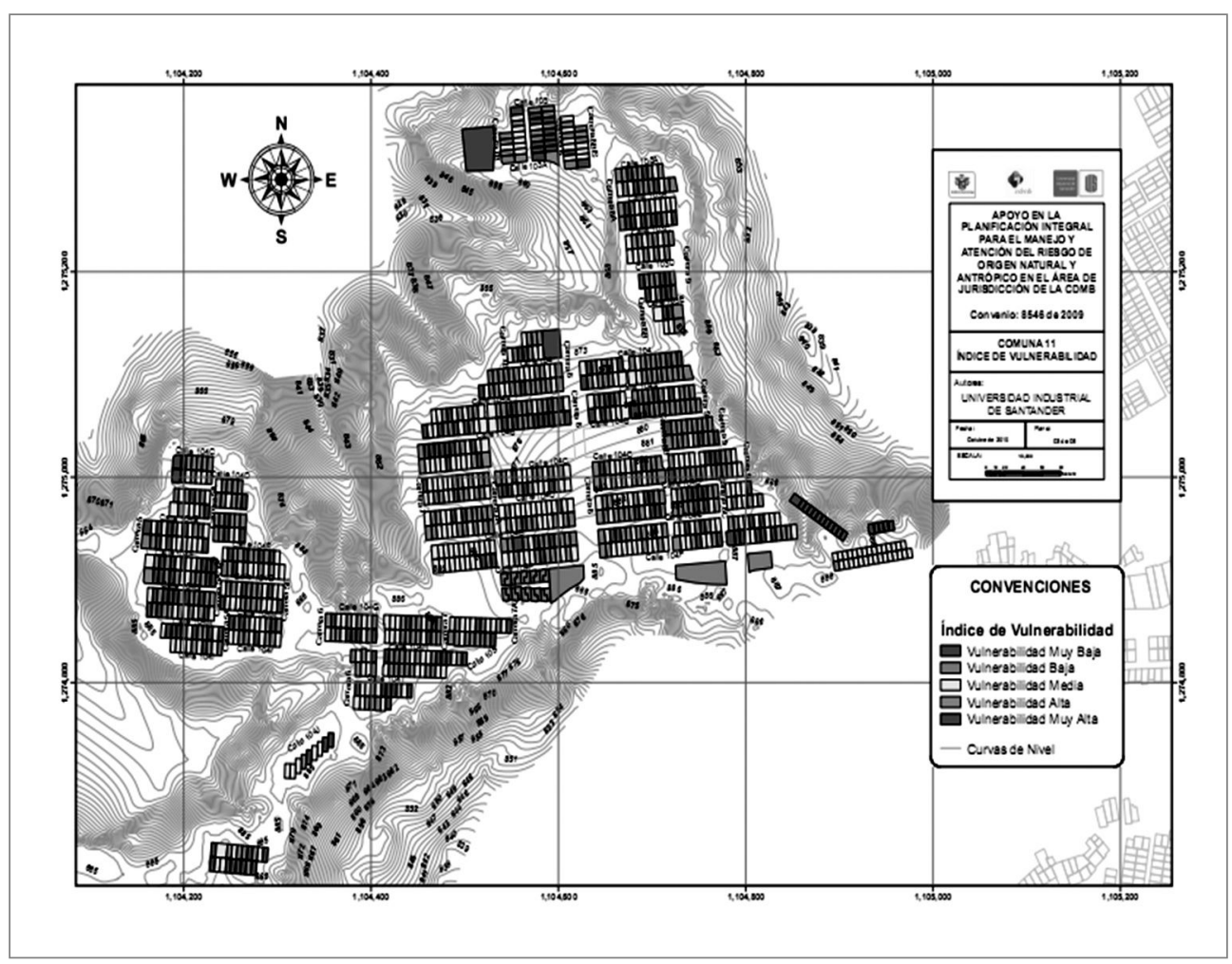

Figura 3. Mapa de vulnerabilidad de la zona de estudio

Figure 3. Vulnerability mapping of the area under study 
The vulnerability index mapping shows that $19.73 \%$ of houses are qualified with a very low vulnerability index; these are one or two-storey houses with a square structural system in every storey. Then $\mathbf{2 5 . 9 5} \%$ of buildings were qualified with low vulnerability index. $36.49 \%$ of them were registered with a medium vulnerability index. A high vulnerability index was assigned to $16.22 \%$ and finally $1.62 \%$ with a very high vulnerability index in the whole sample. The latter are located in the neighborhood south-east sector and the high degree of vulnerability is explained by their proximity to the slope.

\section{Conclusions}

Facing the current need of conducting vulnerability studies due to several nature threads, it is a common practice to apply the proposed models on other contexts and to build own models. By applying existing models created from other locations data, where structural characteristics are different, it exposes us to the risk of using an inadequate model and consequently reaching wrong results. On the other hand, creating an own building vulnerability model, for different cities in our nation, demands accurate information about damages in past events or structural experimental behavior records, which are often not available. Therefore, data collection and information analysis due to landslides must be broadened. Hence, the research on building structural behavior shall be strengthened. While this process takes place, it is necessary to develop new models, by using the existing resources. Therefore, the alternative of using expert opinions for these kinds of studies is quite appropriate.

In the case of cities vulnerability such as Bucaramanga, where there are no damage records on masonry buildings either experimental information, the application of the proposed model proved to be simple and practical; therefore, its use is appropriate for studies at great scale. It is expected that new models will be developed based on further information. 
The proposed methodology is based on the evaluation of parameters that affect at a great extent the building vulnerability faced to landslide effects, which correspond to structural system, quality of strength system, foundation position, type of soil, building location, retaining elements availability, drainage systems, area vegetation, height lay out and preservation conditions of the building and surroundings. They may be qualitative or quantitative parameters, but they are defined based on general information of the building.

The parameters chosen for the definition of this model and its respective classifications correspond to buildings' own environmental conditions. Hence, the proposed model is well applicable to cities having similar characteristics to the ones already studied. Besides, vulnerability degree of each parameters classifications and its importance value were defined by means of the use of fuzzy sets theory, which makes the model applicable on places not counting with damage information records either experimental information on study interest areas.

Based on the relation between vulnerability degree of different parameters and their respective importance value, a vulnerability index was defined. This index qualifies structure vulnerability as very low, low, medium, high or very high, by associating it with a numeric value and a representation of response ambiguity.

The method is greatly applicable to high-scale studies, where a great amount of buildings are to be assed by using the minimum possible information. In this way, it allows the comparison among different zones to detect the most vulnerable ones, thus becoming a useful tool for seismic mitigation plans and land-use planning.

The proposed model has been included in the vulnerability construction functions stated by the Planning support project for natural sources and man-induced risks - handling and care - in the jurisdiction area of CDMB (UIS and CDMB, 2011).

\section{Acknowledgements}

The authors are thankful for the support received from Universidad Industrial de Santander (UIS), Bucaramanga Plateau Defense Association (CDMB) 
and Bucaramanga City Hall, during the development of research study titled Planning support project for natural sources and man-induced risks - handling and care - in the jurisdiction area of CDMB (UIS and CDMB, 2011).

\section{Referencias / References}

Bandemer H., Gottwald S. (1996), Fuzzy sets, fuzzy logic, fuzzy Methods with Applications, Jhon Wiley and sons, New York. Chakroborty P., Kikuchi S. (1990), Application of Fuzzy Set Theory to the Analysis of Capacity and Level of Service of Highways, Proceedings of the First International Symposium on Uncertainty Modeling and Analysis, Maryland, USA. Chao R., Ayyub B. (1995), Distributions with Fuzziness and Randomness, Proceedings of ISUMA-NAFIPS'95.

Finlay P.J., Mostyn G.R., Fell R. (1997), Vulnerability to landsliding, Quarterly Journal of Engineering Geology, Geotechnical Society, Toronto, Canada, 1, 307-324.

Leone F., Aste J.P., Leroi E. (1996), Vulnerability assessment of elements exposed to mass-moving: working toward a better risk perception, In: Senneset, K. (Ed.), Landslides. Balkema, Rotterdam, 263-269.

Maldonado E., Chio G., Gómez I. (2007), Índice de vulnerabilidad sísmica en edificaciones de mampostería basado en la opinión de expertos, Ingeniería y Universidad. Volumen 11, no. 2, 149-168.

Suarez J. (2009), Deslizamientos, Técnicas de remediación. Volumen 2, División de Publicaciones UIS, Bucaramanga. Tee A.B., Bowman M.D., Sinha K.C. (1988), A fuzzy mathematical approach for bridge condition evaluation, Civil Engineering System, 5, 17-24.

UIS y CDMB (2011), Apoyo en la planificación para el manejo y atención del riesgo de origen natural y antrópico en el área de jurisdicción de la CDMB, Convenio interadministrativo No 115-2009, suscrito entre la CDMB, la Alcaldía de Bucaramanga y la UIS.

Uzielli M., Nadim F., Lacasse S., Kaynia A.M. (2008), A conceptual framework for quantitative estimation of physical vulnerability to landslides, Engineering Geology, 102, 251-256.

Zadeh L. A. (1965), Fuzzy sets, Information and control. Volumen 8. 